\title{
Axillary Dissection Can Be Avoided in the Majority of Clinically Node-Negative Patients Undergoing Breast-Conserving Therapy, by Dengel et al.
}

\author{
Judy C. Boughey, MD \\ Department of Surgery, Mayo Clinic, Rochester, MN
}

Since the presentation of the American College of Surgeons Oncology Group (ACOSOG) Z0011 clinical trial results at the American Surgical Association 2010 meeting and the American Society of Clinical Oncology (ASCO) 2011 meeting with subsequent publications in Annals of Surgery and the Journal of the American Medical Association (JAMA), breast surgeons around the United States and indeed worldwide have been considering how to incorporate the results of this study into their clinical practice. $^{1,2}$ Many breast surgeons are now forgoing axillary lymph node dissection (ALND) in selected patients undergoing breast conservation surgery (BCS) with one or two positive sentinel lymph nodes (SLN). There remains debate about what proportion of the clinical practice is subject to benefit from the Z0011 results.

Even prior to the presentation of the Z0011 results, breast surgeons in the United States were increasingly avoiding ALND for those patients diagnosed with microscopic disease in the SLNs as documented both in the National Cancer Database (NCDB) as well as the Surveillance Epidemiology and End Results Registry (SEER). ${ }^{3,4}$ In the NCDB, use of SLN only in patients with micrometastatic disease increased from $25 \%$ in 1998 to $45 \%$ in 2005 and similarly in SEER from $21 \%$ in 1998 to $38 \%$ in 2004.

Most recently, the results of the International Breast Cancer Study Group (IBCSG) trial 23-01, which also randomized patients with positive SLNs to ALND versus no ALND, were published. ${ }^{5}$ The IBCSG 23-01 study

(C) Society of Surgical Oncology 2013

First Received: 18 July 2013;

Published Online: 22 August 2013

J. C. Boughey, MD

e-mail: boughey.judy@mayo.edu limited enrollment to patients with isolated tumor cells or micrometastatic disease; however, it did allow patients undergoing $\mathrm{BCS}$ or mastectomy. Interestingly, in the IBCSG 23-01 study design, 6681 patients were registered preoperatively and only 934 patients were randomized based on the operative findings. Thus, only $14 \%$ of registered breast cancer patients met the inclusion criteria of this study. Unfortunately, the paper does not disclose the reasons for the $86 \%$ not randomized, which is presumably mainly accounted for by node-negative patients and patients with positive SLNs with macrometastatic disease.

In the manuscript entitled "Axillary Dissection Can Be Avoided in the Majority of Clinically Node-Negative Patients Undergoing Breast-Conserving Therapy," ${ }^{6}$ the breast surgeons from Memorial Sloan-Kettering Cancer Center (MSKCC) describe their practice since adopting the Z0011 results. Over the 27-month time period from August 2010 to November 2012, they treated a total of 2,157 patients with invasive breast cancer who elected to undergo BCS for a clinical T1 or T2 breast cancer. Only 380 patients $(17.6 \%)$ were actually found to have histologic nodal metastasis and were therefore candidates for consideration of Z0011 results. Of these 380 patients, $93 \mathrm{did}$ not meet Z0011 criteria because of SLN metastasis detected by immunohistochemistry only, conversion to mastectomy, or use of neoadjuvant chemotherapy. An additional 45 patients did not meet Z0011 criteria for axillary preservation (avoidance of ALND) because of the presence of metastases in more than two sentinel nodes, matted nodal disease, or extracapsular extension. Thus, overall 242 patients were able to avoid ALND as a result of incorporation of Z0011 results into the clinical practice. The authors report that ALND was avoided in $84 \%$ of patients undergoing BCS (242 of 287). 
However, if we take these results and look at them the other way around, of the total 2,157 patients with invasive breast cancer undergoing BCS, 242 women avoided ALND as a result of the Z0011 results. In essence, therefore, Z0011 resulted in a management change in these 242 patients, which makes up $11.2 \%$ of all patients undergoing BCS and, obviously, an even smaller percentage of all breast cancer patients, if mastectomy cases are included in the overall equation. So on one hand, this data can be read to show that Z0011 led to avoiding ALND in $84 \%$ of patients having BCS as stated in this manuscript or, alternatively, that Z0011 altered patient management and avoided ALND in $11 \%$ of patients undergoing BCS. The real answer is probably somewhere in between these percentages, as if we look at all 380 patients with histological nodal metastasis and undergoing BCS, the axilla was conserved in 242, which is $63.7 \%$ of all BCS patients with nodal metastases. The authors do allude to the fact that patients with immunohistochemistry (IHC)-detected nodal positivity were excluded, and it is likely that these axillas were also conserved; so including the 58 patients with IHC positive disease would result in axillary preservation in 300 patients $(78.9 \%$ of all BCS patients with nodal metastases).

The proportion of patients eligible for axillary conservation will vary between practices. Practices around the country vary in terms of rates of BCS versus mastectomy, and currently the majority of axillary conservation in most practices is limited to patients undergoing BCS. A total of 11 patients were excluded as a result of the use of neoadjuvant chemotherapy in this series, indicating that $2.9 \%$ of the 380 patients with a positive SLN had received neoadjuvant chemotherapy. Across other centers, rates of use of neoadjuvant chemotherapy will vary and this will also impact applicability of Z0011 results.

Use of preoperative axillary ultrasound with fine needle aspiration (FNA) biopsy of morphologically abnormal lymph nodes was not applied at MSKCC in the timeframe of this study. Additionally in those cases where an ultrasound had been performed elsewhere with a percutaneous biopsy, a repeat ultrasound was used and SLN surgery was still performed if fewer than three abnormal lymph nodes were visualized. Unfortunately, data on the subgroup of patients that were biopsy-proven node positive and treated with SLN biopsy is not fully described in this manuscript, but would be of great interest. Many centers still routinely use preoperative axillary ultrasound with FNA biopsy to evaluate the nodal status preoperatively. This does allow for enhanced discussion with the patient regarding Z0011 and its impact on their surgical management and also can be used for consideration of neoadjuvant chemotherapy especially in those patients with aggressive tumor biology such as triple negative or HER2 positive disease. At Mayo
Clinic Rochester, we reviewed 1,140 T1/T2 breast cancers from January 2007 to December 2010 treated with SLN biopsy. All of these patients were clinically node negative by preoperative axillary ultrasound with or without FNA biopsy, and a total of 144 patients $(13 \%)$ were found to be node positive with the use of SLN biopsy. This node positive rate of $13 \%$ is lower than the $17.6 \%$ nodal positivity seen in the MSKCC study and is likely reflective of having excluded patients with percutaneous FNA biopsy nodal positivity. ${ }^{7}$ Additionally, axillary ultrasound with preoperative ultrasound-guided percutaneous needle biopsy has been shown to streamline surgical care for $29 \%$ of node positive patients, and two-thirds of patients with multiple axillary suspicious lymph nodes on axillary ultrasound had more than two positive lymph nodes, suggesting that they would fall outside of the Z0011 guidelines and should undergo ALND without SLN biopsy. ${ }^{8}$

Clearly the ACOSOG Z0011 study has impacted clinical practices around the country, and the manuscript from the MSKCC team shows how uniform application of its recommendations over a 27 -month period was able to alter the surgical management of 242 patients. This serves as a strong example of how the results of contemporary clinical trials designed to address important clinical questions can be effective in altering patient care. It is encouraging to see that the results of the Z0011 study, which were initially published in 2010-2011, have been able to change and impact the clinical management of patients from 2010 through 2012 and alter day-to-day patient management in centers across the country.

\section{REFERENCES}

1. Giuliano AE, McCall L, Beitsch P, Whitworth PW, Blumencranz $\mathrm{P}$, Leitch AM, et al. Locoregional recurrence after sentinel lymph node dissection with or without axillary dissection in patients with sentinel lymph node metastases: the American College of Surgeons Oncology Group Z0011 randomized trial. Ann Surg. 2010;252:426-32, discussion 432-3.

2. Giuliano AE, Hunt KK, Ballman KV, Beitsch PD, Whitworth PW, Blumencranz PW, et al. Axillary dissection vs no axillary dissection in women with invasive breast cancer and sentinel node metastasis: a randomized clinical trial. JAMA. 2011;305:56975 .

3. Bilimoria KY, Bentrem DJ, Hansen NM, Bethke KP, Rademaker AW, Ko CY, et al. Comparison of sentinel lymph node biopsy alone and completion axillary lymph node dissection for nodepositive breast cancer. J Clin Oncol. 2009;27:2946-53.

4. Yi M, Giordano SH, Meric-Bernstam F, Mittendorf EA, Kuerer HM, Hwang RF, et al. Trends in and outcomes from sentinel lymph node biopsy (SLNB) alone vs. SLNB with axillary lymph node dissection for node-positive breast cancer patients: experience from the SEER database. Ann Surg Oncol. 2010;17(Suppl 3):343-51.

5. Galimberti V, Cole BF, Zurrida S, Viale G, Luini A, Veronesi P, et al. Axillary dissection versus no axillary dissection in patients 
with sentinel-node micrometastases (IBCSG 23-01): a phase 3 randomised controlled trial. Lancet Oncol. 2013;14:297-305.

6. Dengel L, Van Zee KJ, King TA, et al. Axillary dissection can be avoided in the majority of clinically node-negative patients undergoing breast-conserving therapy. Ann Surg Oncol. doi:10. 1245/s10434-013-3200-6.

7. Ibrahim-Zada I, Grant CS, Glazebrook KN, Boughey JC. Preoperative axillary ultrasound in breast cancer: safely avoiding frozen section of sentinel lymph nodes in breast-conserving surgery. $J$ Am Coll Surg. 2013;217:7-15.

8. Hieken TJ, Trull BC, Boughey JC, et al. Preoperative axillary imaging with percutaneous lymph node biopsy is valuable in the contemporary management of breast cancer patients. Surgery. (Accepted for publication). 